\title{
Torque Measurement Device Using an Integrated Microdisplacement Sensor
}

\author{
Takuma Iwasaki*, Toshihiro Takeshita, Yuji Arinaga ${ }^{1}$ and Renshi Sawada \\ Graduate School of Systems Life Sciences, Kyushu University, \\ 744 Motooka, Nishi-Ku, Fukuoka 819-0395, Japan \\ ${ }^{1}$ Corporate R \& D Center, Yaskawa Electric Corporation, 12-1, Otemachi, Kokurakita-ku, \\ Kitakyushu 803-8530, Japan
}

(Received June 12, 2013; accepted August 22, 2013)

Key words: MEMS, robot arm, sensor, torque

In this paper, we propose a torque measurement device embedded in robot arms. The device uses an integrated microdisplacement sensor and trapezoidal frame. The sensor tip of $3 \mathrm{~mm}$ area and $0.7 \mathrm{~mm}$ thickness measures the mirror tilt angle on the underside of the frame caused by a torque applied to the frame. We have successfully measured a linear output change for the torque and found that the gradient of the output change differed largely from the material and the corner angle of the frame.

\section{Introduction}

In recent works, torque sensors have been used to control electro power steering (EPS) in cars to detect torque applied to a steering wheel, where the torque is detected through measurement of a magnetic change in a torsion bar without a contact. ${ }^{(1-3)}$ Magnetictype torque sensors have the advantage of robustness and remarkable resolution, yet they have a weakness associated with electromagnetic noise. ${ }^{(4)}$ There have been other types of torque sensors that exploit strain gauges $^{(5-10)}$ and optics. ${ }^{(4,11)}$ Hashimoto et al. have reported torque sensors using strain gauges to detect the dynamic torque applied to a harmonic drive. ${ }^{(8-10)}$ These torque sensors, which use magnetic change, strain gauges, and optics, are tiny and maintain high accuracy; therefore, they are applicable to robot arms.

The motor-generated torque of a robot arm is calculated as a product of a current and a torque constant. However, robot arms are actually driven by an axial torque output by reducers, and this torque cannot be calculated through a product of the motor-generated torque and a reduction gear ratio since they are unequal owing to the friction generated at the reducers. Therefore, detecting the axial torque output by reducers will directly enable sophisticated control of the robot arms and is essential for developing safety functions for robots that coexist with humans.

${ }^{*}$ Corresponding author: e-mail: iwasakitakuma.nanomicro416@gmail.com 
There are three approaches to detect axial torque output by reducers: disturbance observer, a wrist 6-axis force sensor, and an articular torque sensor. A disturbance observer is a method that deduces an external unknown or uncertain disturbance torque without the use of an additional sensor. This technique is widely used for industrial robots. ${ }^{(12-14)}$ This method is unable to achieve high accuracy, yet is cheap to implement. A wrist 6-axis force sensor cannot detect external force except that which occurs at the wrist. In comparison, an articular torque sensor can precisely detect a tiny external force, even if the force is applied at a midpoint of an arm. Therefore, the articular torque sensor is better suited for external torque sensing.

In this study, we present a torque measurement device combining a trapezoidal metallic frame with an integrated optical microdisplacement sensor, ${ }^{(15,16)}$ fabricated using MEMS technology. Our sensor can measure linear displacement and the biaxial tilt angle of an object using one vertical-cavity surface-emitting laser (VCSEL) and four photodiodes (PDs), and can be downsized drastically in comparison with the conventional displacement sensor by monolithic fabrication. The VCSEL differs from a normal laser in that the beam is emitted perpendicular to the chip surface; it is superior with respect to lower production costs, power consumption, and integration capabilities. ${ }^{(17)}$ Our torque measurement device has many features. Firstly, our displacement sensor is very small, $3 \mathrm{~mm}^{2}$, and achieves a very high accuracy despite its simple structure. Secondly, we can alter the measurement range and sensitivity by only changing the shape of the frame. The purpose of this study is to show that we can detect torque using our displacement sensor, and compare experimental results in four types of trapezoidal metallic frames.

\section{Displacement Sensor}

Our torque measurement device applies the integrated optical microdisplacement sensor shown in Fig. 1. The structure of the displacement sensor is described in $\S 2.1$. The measurement principle is detailed in $\S 2.2$.

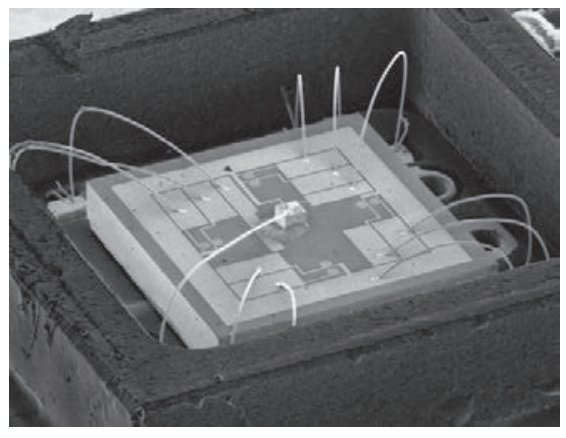

(a)

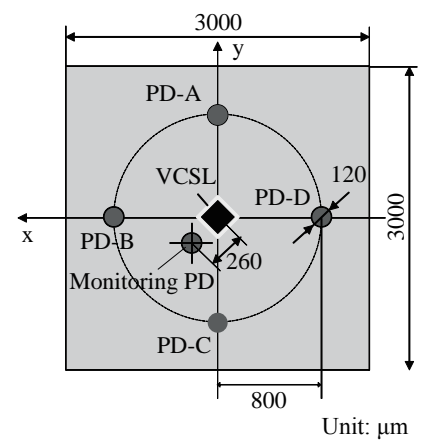

(b)

Fig. 1. View of integrated microdisplacement sensor $\left(3 \times 3 \mathrm{~mm}^{2}\right.$, and $0.7 \mathrm{~mm}$ thickness). (a) Photograph of sensor tip and (b) schematic view of sensor structure. 


\subsection{Structure}

The optoelectronic part of the sensor is $3 \times 3 \mathrm{~mm}^{2}$ with a thickness of $0.7 \mathrm{~mm}$. As shown in Fig. 1, a VCSEL in the center of the sensor and four detecting PDs, which provides electrical current according to the intensity at the PD, are integrated monolithically and arranged in a concentric pattern. The diameter of each PD is 120 $\mu \mathrm{m}$ and the distance from the center of the VCSEL to the center of each outer PD is 800 $\mu \mathrm{m}$. In addition, a monitoring PD is located near the VCSEL at a distance of $260 \mu \mathrm{m}$. This assembly is coated with glass as shown in Fig. 2. There is a reflective area near the center of the glass as shown. Some of the emitted beam from the VCSEL enters the monitoring PDs after being reflected from the reflective region. The sensor can utilize the reflection to obtain feedback of the laser output change.

\subsection{Principle of operation}

The sensor can measure linear displacement and tilt of an object using the VCSEL, four detecting PDs, and an external mirror attached to the object. The VCSEL emits beams in a direction perpendicular to the sensor surface, with a specified spread angle. Figure 3 shows a side view of the sensor and mirror, along with the corresponding reflected beam distributions on the sensor. We illustrate the results we achieved in the following sections by changing the distance between the mirror and the sensor, and the tilt angle of the mirror with an automatic translation stage.

\subsubsection{Linear displacement}

As the distance between the mirror and the sensor is varied, the output of the PDs changes as shown in Figs. 3(a) and 3(b). Figure 4 shows the relationship between the distance and the output $S$, which is given as

$$
S=P_{\mathrm{A}}+P_{\mathrm{B}}+P_{\mathrm{C}}+P_{\mathrm{D}},
$$

where $P_{\mathrm{A}}-P_{\mathrm{D}}$ are the outputs of PD-A - PD-D, respectively.
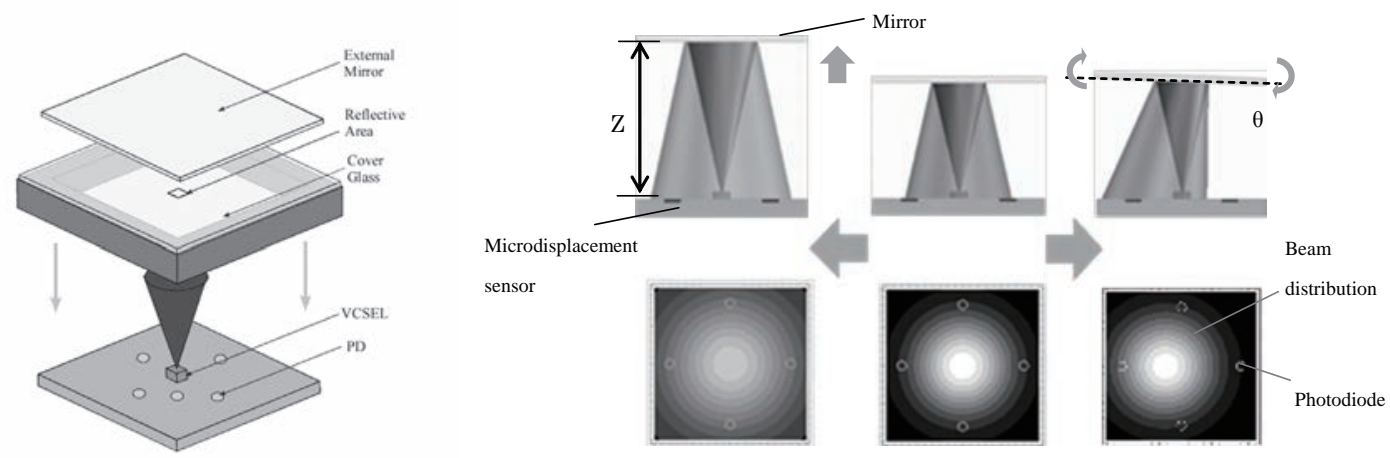

(a)

(b)

(c)

Fig. 2 (left). Overall view of structure of displacement sensor that measures linear displacement and biaxial tilt angle with one VCSEL, four PDs, and a mirror.

Fig. 3 (right). View from side of sensor and beam distribution on sensor chip: (a) linear displacement, (b) default position, and (c) tilt. 


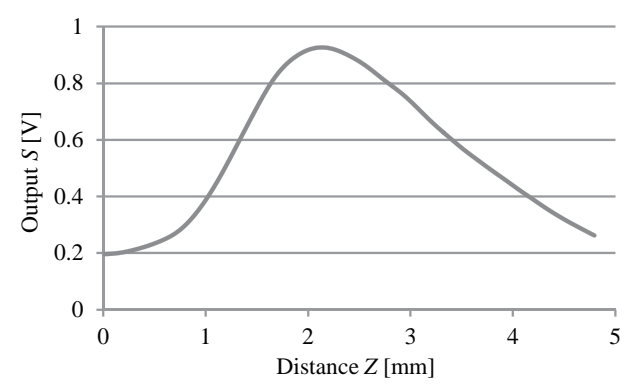

Fig. 4. Relationship of output $S$ and distance $Z$ between mirror and sensor.

When the mirror is close to the sensor, the distribution is small, and only a small amount of the reflected beam enters the PDs. As a result, the output $S$ is also small. As the mirror moves away from the sensor, the laser beam distribution spreads and the PD areas that receive the beam increase proportionately. Therefore, the output $S$ increases linearly and peaks when the beam completely enters the PDs. As the distance increases further, the output $S$ decreases linearly as the intensity weakens. The sensor can be used to measure the linear displacement through the use of the two linear regions of the graph.

\subsubsection{Tilt angle}

If we modify the tilt angle of the mirror, the direction of the reflected beam distribution is changed, as shown in Figs. 3(b) and 3(c). This causes a difference in the PD intensity. Figure 5 shows the relationship of the tilt angle and output $S$ when the distance between the sensor and the mirror is $2 \mathrm{~mm}$. The output can be expressed as

$$
\begin{gathered}
S_{\mathrm{x}}=\frac{P_{\mathrm{A}}-P_{\mathrm{C}}}{P_{\mathrm{A}}+P_{\mathrm{C}}}, \\
S_{\mathrm{y}}=\frac{P_{\mathrm{B}}-P_{\mathrm{D}}}{P_{\mathrm{B}}+P_{\mathrm{D}}} .
\end{gathered}
$$

In the case of $\mathrm{x}$ - or y-axis rotations, (2) or (3) is used, respectively.

For example, when the mirror tilts clockwise as in Fig. 3(c), the intensity of PD-B increases and that of PD-D decreases. Therefore, the output $S_{\mathrm{y}}$ increases. We can determine the tilt angle using the region where $S$ changes linearly.

\section{Design}

Our torque measurement device uses the displacement sensor described in the previous subsection. The displacement sensor is covered with a trapezoidal frame, where the mirror is attached to the underside of the frame. When torque is applied to the frame, the frame will be deformed and the mirror tilts as shown in Fig. 6. The torque can be detected by measuring the tilt angle of the mirror with the displacement sensor. In Fig. 7 , the location where the torque measurement device is embedded is shown. The central axis does not rotate and external torque is applied to the outside of the wheel. 


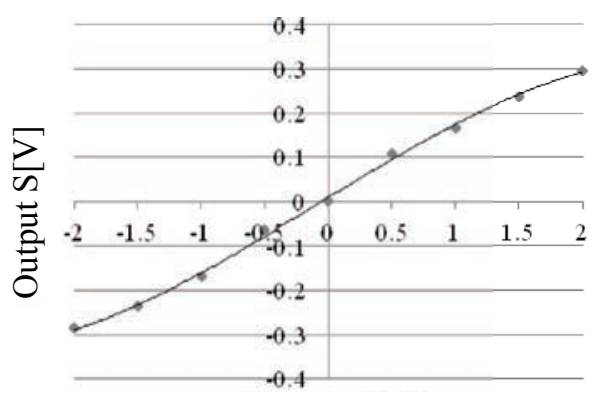

Tilt angle $\theta\left[^{\circ}\right]$

Fig. 5. Relationship between output and mirror tilt angle.

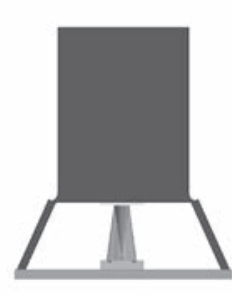

(a)

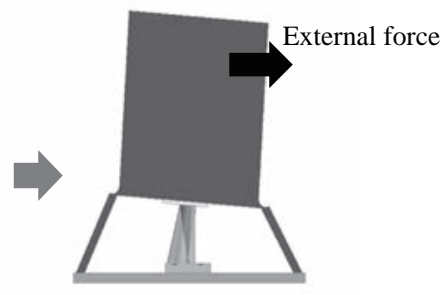

(b)

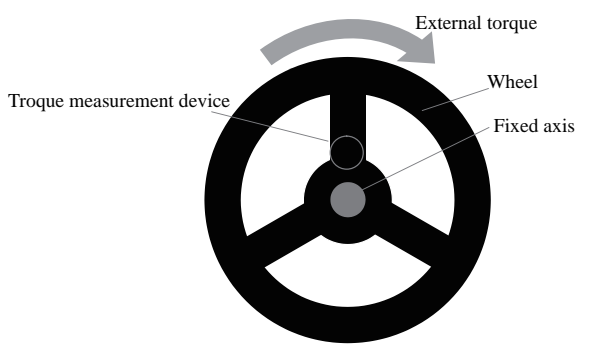

Fig. 6 (left). Conceptual diagram of torque measurement. (a) Default position and (b) when torque is applied.

Fig. 7 (right). Conceptual diagram of torque position of measurement device.

\section{Experiment}

To ensure that the torque can be measured using the principle mentioned in the previous sections, we conducted an experiment using four types of trapezoidal metallic frame(s) as shown in Fig. 8 and Table 1. Figure 9 shows an experimental system. The displacement sensor is fixed to a board and covered by the frame. An artificial torque is applied to the frame through the attached object, which is pulled with weights through a pulley. An external force was applied to the point $10 \mathrm{~mm}$ above the frame surface. The driving current of the VCSEL was $0.008 \mathrm{~A}$ and the driving voltage of the amplifier of PDs was $5.00 \mathrm{~V}$. We applied a torque through the attached weights, varying them from 0 to $3.0 \mathrm{~kg}$ on each frame. The gravity acceleration was fixed as $9.806 \mathrm{~m} / \mathrm{s}^{2}$, and the distance between the surface of the frame and the working point of the force was 10.0 $\mathrm{mm}$; therefore, the applied torque ranged from 0 to $0.294 \mathrm{Nm}$. Since our copper frames were likely to collapse at the maximum torque possible, we only allowed the weight to range up to $2.0 \mathrm{~kg}$, that is, $0.196 \mathrm{~N}$. 


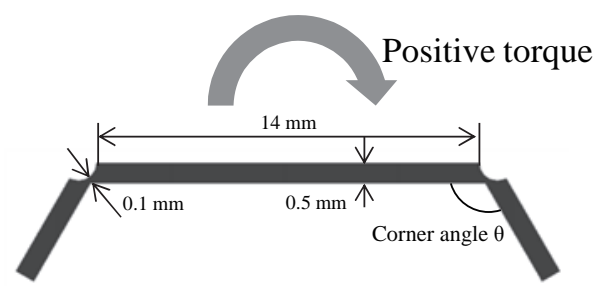

Fig. 8. Design of frame.

Table 1

Material and shape of each frame.

\begin{tabular}{cccccc}
\hline & \multirow{2}{*}{ Material } & \multirow{2}{*}{ Young's modulus } & \multicolumn{3}{c}{ Corner angles $\theta$} \\
\cline { 4 - 6 } & & & Left & Right & Average \\
\hline a & Stainless Steal & $197 \mathrm{GPa}$ & $105^{\circ}$ & $110^{\circ}$ & $107.5^{\circ}$ \\
b & (SUS304) & $197 \mathrm{GPa}$ & $115^{\circ}$ & $120^{\circ}$ & $117.5^{\circ}$ \\
c & Copper (OFC) & $128 \mathrm{GPa}$ & $111^{\circ}$ & $112^{\circ}$ & $111.5^{\circ}$ \\
$\mathrm{d}$ & Copper (OFC) & $128 \mathrm{GPa}$ & $118^{\circ}$ & $122^{\circ}$ & $120^{\circ}$ \\
\hline
\end{tabular}

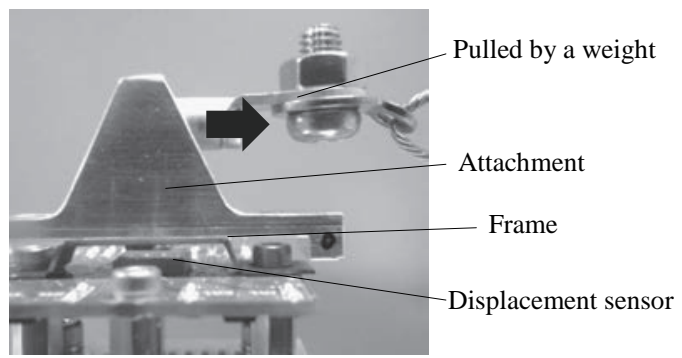

Fig. 9. Photograph of experimental system.

In Fig. 10, the experimental results are shown. The output increases linearly with torque for all types of frames. In addition, the gradient of the output change depended largely on the shape and material of the frames. The measured gradients of the frames with a larger corner angle (triangle points) were steeper than those of the frames with smaller corner angles (round points) with both materials and torque directions. However, some of the frames indicated a difference in the gradient in the direction of the torque.

\section{Discussion}

This measurement device is expected to detect the magnitude and direction of torque using the linear output changes. In addition, we can change the sensitivity and measurement range by altering the material and corner angle of the frame.

We found that the gradient of the output value change is related largely to the stiffness, that is, the material and shape, of the frame. It is widely known that the 


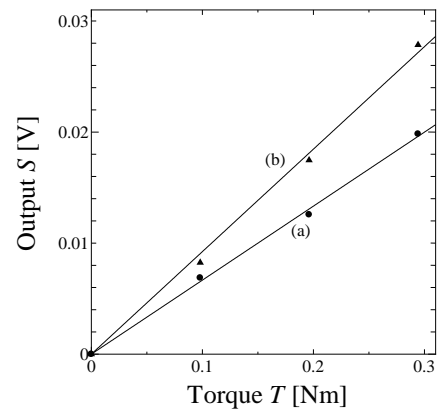

(a)

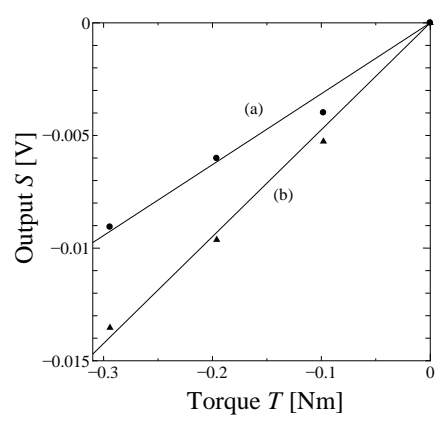

(c)

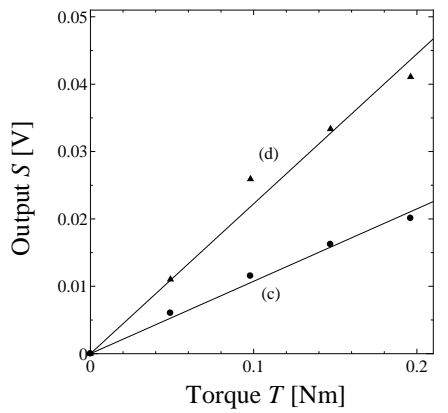

(b)

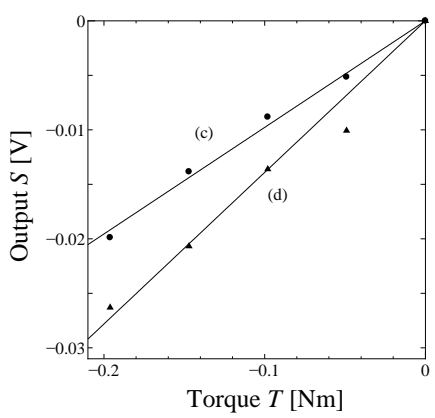

(d)

Fig. 10. Relationship of torque $T$ and output $S$. (a) and (b) Stainless steal. (c) and (d) Copper frame.

copper frames would be easier to deform than stainless steel frames. The frames also became easier to deform by increased torque as the corner angle of the frame widened. As expected, the copper frames have a steeper gradient in the output change than the stainless steel frames. The frames with a larger corner angle also have a larger change in output value than the frame with a smaller corner angle. Hence, we can change the sensitivity and measurement range of the sensor by altering the material and shape of the frame covering the displacement sensor. For example, it is better to use the frame that is hard to deform when we want to measure a relatively large torque.

There is a small difference between the angle of the left corner and that of the right corner as shown in Table 1, which may explain the possible cause of the difference in the gradient in the direction of the torque. The gradient becomes steeper as the corner angle widens; however, the change in output values observed when a positive torque was applied to the right corner with an angle of $110^{\circ}$ (round points in the graph of stainless steel) was larger than when a negative torque was applied to the left corner with an angle of $115^{\circ}$ (triangle points in the graph of stainless steel). Thus, it could be inferred that the gradient of the output change depends on both the corner angles of the left and right sides of the frame. 
In this experiment, we applied a torque up to $0.3 \mathrm{Nm}$. However, a torque sensor is requested to measure up to $100 \mathrm{Nm}$ of maximum output torque ${ }^{(5)}$ when it is used in a joint unit. Therefore, we need to increase the stiffness of the frame in future work.

\section{Conclusions}

In this paper, we have proposed a torque measurement device and succeeded in obtaining a linear output change for torque by combining a metallic trapezoidal frame, which has a mirror on the underside, and an integrated microdisplacement sensor. The sensor is fabricated using MEMS technology and has an area of $3 \mathrm{~mm}^{2}$ with a thickness of $0.7 \mathrm{~mm}$. The device detects torque by measuring a tilt angle of the mirror with one VCSEL and four detecting PDs. In addition, we have succeeded in changing the gradient of the output measurement by altering the material and the corner angle of the frame. As a result, we could measure a larger torque by designing an appropriate frame in order to embed it in robot arms.

\section{References}

1 K. Yoshida: Koyo Eng. J. 160 (2001) 62.

2 Y. Sakai and T. Kaoku: Koyo Eng. J. 167 (2005) 61.

3 S. Ouyang, J. Murakami and K. Kato: Hitachi Cable Rev. 26 (2007) 41.

4 D. Tsetserukou, R. Tadakuma, H. Kajimoto and S. Tachi: Proc. IEEE Int. Conf. on Robotics nad Automation, 2006, p. 1674.

5 M. Hashimoto, Y. Kiyosawa and R. P. Paul: IEEE Trans. Robot. Autom. 1 (1993) 108.

6 T. Kawakami, K. Ayusawa, H. Kaminaga, C. Ott and Y. Nakamura: Proc. JSME Conf. Robot. Mechatron., 2008.

7 D. Vischer and O. Khatib: IEEE Trans. Robot. Autom. 4 (1995) 537.

8 M. Hashimoto and I. Godler: J. Rob. Soc. Jpn. 5 (1997) 802.

9 I. Godler and M. Hashimoto: Proc. Ann. Conf. of the IEEE (1998) 1818.

10 I. Godler, M. Hashimoto and M. Horiuchi: SI2001, 2001, 1A1_45.

11 S. Shams, D. Shin, J. Han, J. Y. Lee, K. Shin and C. Han: IEEE/RSJ Int. Conf. on Intelligent Robots and Systems (2011) 5127.

12 W.-H. Chen, D. J. Ballance, P. J. Gawthrop and J. O’Reilly: IEEE Trans. Ind. Electron (2000) 932.

13 C. J. Kempt and S. Kobayashi: IEEE Trans. Contr. Syst. T 5 (1999) 513.

14 G. Zhang and J. Furusho: J. JSME. 592 (1995) 4687.

15 T. Takeshita, Y. Peng, N. Morita, H. Ando, E. Higurashi and R. Sawada: Joint Int. IMEKO Symp. (2011) urn:nbn:de:gbv:ilml-2011imeko-015:6.

16 I. Itaru, R. Sawada, E. Higurashi, S. Sanada and D. Chino: Ssens. Actuators, A 2 (2007) 269.

17 R. Paschotta: "Pr Photonics Encyclopedia" http://www.rp-photonics.com/vertical_cavity_surf ace_emitting_lasers.html (2013/03/27). 